\title{
Evolution of Dynamic Loads in Steel Spur Gears
}

\author{
S. Amaldhasan ${ }^{1 *}$ and S. PonPaul ${ }^{2}$ \\ 1,2 Department of Mechanical Engineering, Bharath Institute of Science and Technology, Bharath University, \\ Chennai-600073,Tamilnadu; amal_as@rediffmail.com', ponpaul_ranjith@yahoo.com²
}

\begin{abstract}
The Complete study of Literature and reporting on how Spur gears are designed using steel-steel alloys and dynamic load is calculated for the spur gears. The analytical model is developed to simulate the load sharing characteristics through a mesh cycle. The model takes into account the main internal factors of dynamic load as time-varying mesh stiffness and composite tooth profile errors. Comparative different types of dynamic load processors study are included, which shows the different processors of the dynamic load. Pro $\mathrm{E}$ is an interactive CAD/CAM system, a fully 3-D double precession system that allows accurate description of almost any geometric shape. Pro E modeling provides capabilities to help the design engineer to perform conceptual and detailed deigns. It is a feature and constrained based solid modeler that allows users to create and edit complex solid models interactively. MSC Nastran is a powerful general purpose, Finite element analysis solution for small to complex assemblies. A standard tool, in the field of structural analysis for over four decades, Nastran provides a wide range of analysis capabilities including linear, static's, dynamic, displacement, strain, stress, vibration, heat transfer and more. Nastran can handle any material type from plastic and metal to composites and hyper elastic materials.
\end{abstract}

Keywords: Pro E , MSC Nastran, Spur Gears.

\section{Introduction}

Any toothed member designed to transmit motion to another one, or received motion from it, by means of successively engaging tooth is called a (toothed) gear. Gears are a means of changing the rate of rotation of a machinery shaft. They can also change the direction of the axis of rotation and can change rotary motion to linear motion. Gears are of several categories and can be combined in a multitude of ways, some of which are meshing circular spur gears, rack and pinion spur gears, and worm gears. Helical and herringbone gears utilize curved teeth for efficient, high-capacity power transmission. Worm gears, driven by worms transmit motion between non-intersecting rightangle axes. Gears mate or mesh via teeth with a very specific geometry. Gears are used for two basic purposes; increase or decrease of rotation speed and increase or decrease of power or torque. Torque is a measure of a force to produce torsion and rotation about an axis. To increase speed and reduce torque a large drive gear is coupled to a smaller driven gear.

\subsection{Gear Manufacturing Materials}

Mild steel is a poor material for gears as it has poor resistance to surface loading. The carbon content for unhardened gears is generally $0.4 \%(\mathrm{~min})$ with $0.55 \%(\mathrm{~min})$ carbon for the pinions. Dissimilar materials should be used for the meshing gears - this particularly applies to alloy steels. Alloy steels have superior fatigue properties compared to carbon

*Corresponding author:

S. Amaldhasan (amal_as@rediffmail.com) 
steels for comparable strengths. For extremely high gear loading case hardened steels are used the surface hardening method employed should be such to provide sufficient case depth for the final grinding process used. While selecting a gear material depends on its cost, strength, wear resistance, noise level etc. has to be compromised. Materials such as ferrous metals Non-Ferrous metals, Non metals.

\subsection{Law of Gearing}

In order for two meshing gearing to maintain a constant angular velocity ratio, they must satisfy the fundamental law of gearing. The shape of the teeth of a gear must be such that the common normal at the point of contact between two teeth must always pass through a fixed point on the line of centers. Conjugate action on spur gears: - The gear-tooth profile that will transmit through each other uniform rotary motion. The action between such teeth is called conjugate gear-tooth action

\section{Literature Review}

The principal advances in the engineering of gears have been in measurement, inspection and manufacturing techniques. These, and a new lubrication theory, have contributed to the improved performance required from modern high-speed transmissions. Unfortunately, gear case design has not kept up with the design of gears themselves [1]. In a theoretical analysis of a single pair of high-class gears three internal sources of vibration are considered. Dynamic tooth forces and amplitudes of vibration were found from photo-elastic stress patterns of model gears with both small and large errors [2]. Spur gear tooth force analysis, Spur gear - tooth stresses, Tooth bending stress Lewis equation, Tooth bending stress - AGMA procedure, Bending fatigue strength - AGMA procedure, Permissible bending stress, Buckingham equation for dynamic load on gears [3]. The model takes into account the main internal factors of dynamic load as time- varying mesh stiffness and composite tooth profile errors. The complicated phenomenon of contact tooth pairs alternation during mesh cycle is integrated in this dynamic load modeling [4].

\section{Spur Gear Design}

The formulae and parameters are used from a Hand Book of Gear Design by G.M.Maitra and Machine Design Data Book - P.S.G. College of Technology.

\subsection{Dimensions for Standard Spur Gear}

Pitch circle diameter for pinion, $d_{1}=m^{\star} z_{1} m m$ Pitch circle diameter for Gear, $\mathrm{d}_{2}=\mathrm{m}^{*} \mathrm{z}_{2} \mathrm{~mm}$ Base circle diameter for Pinion, $d_{b 1}=d 1^{*} \cos \alpha m m$ Base circle diameter for Gear, $\mathrm{d}_{\mathrm{b} 2}=\mathrm{d}_{2}{ }^{*} \cos \alpha \mathrm{mm}$ Tip circle diameter for pinion, $\mathrm{d}_{\mathrm{a} 1}=\mathrm{d}_{1}+2 \mathrm{mmm}$ Tip circle diameter for gear, $\mathrm{d}_{\mathrm{a} 2}=\mathrm{d}_{2}+2 \mathrm{mmm}$ Root circle diameter for pinion, $\mathrm{d}_{\mathrm{f} 1}=\mathrm{d}_{1}-2^{\star} 1.25 \mathrm{~mm}$ Root circle diameter for gear, $d_{f 2}=d_{2}-2^{\star} 1.25 \mathrm{~mm}$ Tooth thickness on pitch circle, $S=\pi \mathrm{m} / 2 \mathrm{~mm}$ Clearance $\mathrm{c}=0.25 \mathrm{~mm}$

Centre distance, $a=\left(d_{1}+d_{2}\right) / 2$ or $m\left(z_{1}+z_{2}\right) / 2$

Total Depth, $h=2.25^{\star} \mathrm{m} \mathrm{mm}$

Circular pitch, $p=\pi \mathrm{m} \mathrm{mm}$

Face width, $b=3^{*} \pi^{*} \mathrm{~m} \mathrm{~mm}$

Ratio of gear width to center distance $\psi=b / a$

Gear ratio $\mathrm{i}=\mathrm{z}_{1} / \mathrm{z}_{2}=\mathrm{n}_{1} / \mathrm{n}_{2}=\omega_{1} / \omega_{2}$

Module $\mathrm{m}=\mathrm{d} / \mathrm{z} \mathrm{mm}$

Diametral pitch $\mathrm{p}_{\mathrm{d}}=\mathrm{z} / \mathrm{d}$ or $1 / \mathrm{m}$

Power $\mathrm{P}=2 \pi \mathrm{n}_{1} \mathrm{M}_{\mathrm{t}} / 60 \mathrm{KW}$

Gear life $\mathrm{L}=60 \mathrm{n}_{1} \mathrm{~L}_{\mathrm{H}}$ revolutions

Pinion Torque $M_{t}=P^{\star} 60 /\left(2 \pi n_{1}\right) N$ mm

\subsection{Design Bending Stress}

$\sigma_{\mathrm{b}}=\left(1.4 \mathrm{k}_{\mathrm{bl}} \sigma_{-1}\right) /\left(\mathrm{n}_{\mathrm{\sigma}}\right)$....for rotation in one direction

$\sigma_{\mathrm{b}}=\left(\mathrm{k}_{\mathrm{bl}} \sigma_{-1}\right) /\left(\mathrm{n} \mathrm{k}_{\mathrm{\sigma}}\right)$....... for rotation in both directions

$\mathrm{k}_{\mathrm{bl}}=$ life factor

$\mathrm{k}_{\sigma}=$ fillet stress concentration factor

$\sigma_{-1}=$ endurance limit in reversed bending

$\mathrm{n}=$ factor of safety,

\subsection{Induced Bending Stress}

$$
\sigma_{\mathrm{b}}=\frac{\boldsymbol{i}+1}{\boldsymbol{a m b y}}\left[\mathrm{M}_{\mathrm{t}}\right]
$$

$\mathrm{Y}$ is form factor taken from Ref

\subsection{Design Torque}

$$
\begin{aligned}
& {\left[\mathrm{M}_{\mathrm{t}}\right]=\mathrm{k}_{0}^{*} \mathrm{k}^{*} \mathrm{k}_{\mathrm{d}}{ }^{*} \mathrm{M}_{\mathrm{t}}} \\
& \mathrm{k}_{0}=\text { shock loading condition } \\
& \mathrm{k}=\text { load concentration factor } \\
& \mathrm{k}_{\mathrm{d}}=\text { dynamic load factor } \\
& \mathrm{M}_{\mathrm{t}}=\text { pinion Torque }
\end{aligned}
$$

\subsection{Design Contact Stress}

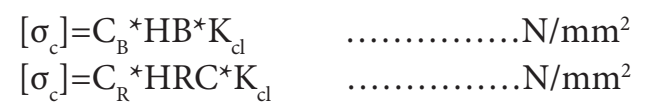


where, $C_{B}$ or $C_{R}$-coefficient depending on the surface hardness, ... Table 3.1

$\mathrm{HB}$ or HRC-Brinell or Rockwell hardness number. $\mathrm{K}_{\mathrm{cl}}$ - life factor,

\subsection{Induced Contact Stress}

$$
\sigma_{c}=0.74 \frac{i+1}{a} \sqrt{\frac{i \pm 1}{i b} E[\mathrm{Mt}]}
$$

$E$-Equivalent Young's modulus $=2 \mathrm{E}_{1} \mathrm{E}_{2} /\left(\mathrm{E}_{1}+\mathrm{E}_{2}\right)$

Pitch line velocity $V=\pi d_{1} n_{1} / 60$

Table 3.1. Proportions of Standard Gear Teeth

\begin{tabular}{lll}
\hline & $20^{\circ}$ Full Depth Involute & $20^{\circ}$ Stub Involute \\
\hline Addendum & $\mathrm{M}$ & $0.8 \mathrm{~m}$ \\
Minimum & $1.25 \mathrm{~m}$ & $\mathrm{M}$ \\
dedendum & & \\
Whole depth & $2.25 \mathrm{~m}$ & $1.8 \mathrm{~m}$ \\
Clearance & $0.25 \mathrm{~m}$ & $0.2 \mathrm{~m}$ \\
\hline
\end{tabular}

Table 3.2. Stress Concentration Factor for Fillet, $\mathrm{K}_{\sigma}$

\begin{tabular}{lc}
\hline Material and Heat Treatment & $\mathrm{K}_{\sigma}$ \\
\hline steel, normalised, surface hardened & 1.5 \\
steel, case hardened (low carbon steels) & 1.2 \\
cast iron & 1.2 \\
\hline
\end{tabular}

Table 3.3. Factor of Safety, N

\begin{tabular}{lllc}
\hline Material & $\begin{array}{l}\text { Mode of } \\
\text { Manufacture }\end{array}$ & Heat Treatment & $\begin{array}{c}\text { Factor of } \\
\text { Safety, } \mathbf{n}\end{array}$ \\
\hline Steel Cast & Cast & $\begin{array}{l}\text { No heat treatment } \\
\text { tempered or }\end{array}$ & 2.5 \\
Iron & & normalised & 2.0 \\
Steel & Cast or forged & Case hardened & 2.0 \\
& Forged & Surface hardened & 2.5 \\
& & Normalised & 2.0 \\
\hline
\end{tabular}

Table 3.4. Life Factor For Surface (Contact Compressive) Strength, $\mathrm{K}_{\mathrm{cl}}$

\begin{tabular}{llll}
\hline Material & $\begin{array}{l}\text { Surface } \\
\text { Hardness }\end{array}$ & $\begin{array}{l}\text { Life In } \\
\text { Number Of } \\
\end{array}$ & $\begin{array}{l}\text { Life Factor } \\
\mathrm{K}_{\mathrm{cl}}\end{array}$ \\
\hline Steel & $\mathrm{HB}$ & $\geq 10^{7}$ & 1 \\
& $\leq 350$ & $<10^{7}$ & $6 \sqrt{ }\left(10^{7} / \mathrm{N}\right)$ \\
& & $\geq 25^{\star} 10^{7}$ & 0.585 \\
& $>350$ & $<25^{\star} 10^{7}$ & $6 \sqrt{ }\left(10^{7} / \mathrm{N}\right)$ \\
Carbon & & $6 \sqrt{ }\left(10^{7} / \mathrm{N}\right)$ \\
\hline
\end{tabular}

Table 3.5. Shock Factor, $\mathrm{K}_{0}$

\begin{tabular}{ll}
\hline Type of load & $\mathrm{K}_{0}$ \\
\hline Steady & 1.0 \\
Light shock & 1.25 \\
Medium shock & 1.5 \\
Heavy shock & 2.0 \\
\hline
\end{tabular}

Table 3.6. Load Concentration Factor, $\mathrm{k}$ for Cylindrical Gears

\begin{tabular}{|c|c|c|c|c|}
\hline \multirow{2}{*}{$\psi_{\mathrm{p}}=\mathrm{b} / \mathrm{d}_{1}$} & \multirow{2}{*}{$\begin{array}{l}\text { Bearing Close } \\
\text { To Gear And } \\
\text { Symmetrical }\end{array}$} & \multicolumn{2}{|c|}{ Asymmetrical } & \multirow{2}{*}{$\begin{array}{l}\text { Over } \\
\text { Hung } \\
\text { Pinion }\end{array}$} \\
\hline & & $\begin{array}{c}\text { Very Rigid } \\
\text { Shaft }\end{array}$ & $\begin{array}{c}\text { Less Rigid } \\
\text { Shaft }\end{array}$ & \\
\hline 0.2 & 1 & 1 & 1.05 & 1.15 \\
\hline 0.4 & 1 & 1.04 & 1.1 & 1.22 \\
\hline 0.6 & 1.03 & 1.08 & 1.16 & 1.32 \\
\hline 0.8 & 1.06 & 1.13 & 1.22 & 1.45 \\
\hline 1.0 & 1.1 & 1.18 & 1.29 & - \\
\hline 1.2 & 1.14 & 1.23 & 1.36 & - \\
\hline 1.4 & 1.19 & 1.29 & 1.45 & _ \\
\hline 1.6 & 1.25 & 1.35 & 1.55 & - \\
\hline
\end{tabular}

Table 3.7. Form Factor, y

\begin{tabular}{cccc}
\hline $\mathrm{Z}$ & $\mathrm{y}$ & $\mathrm{Z}$ & $\mathrm{Y}$ \\
\hline 12 & 0.308 & 35 & 0.452 \\
14 & 0.330 & 40 & 0.465 \\
16 & 0.355 & 45 & 0.471 \\
18 & 0.377 & 50 & 0.477 \\
20 & 0.389 & 60 & 0.490 \\
22 & 0.402 & 80 & 0.499 \\
24 & 0.414 & 100 & 0.505 \\
26 & 0.427 & 150 & 0.515 \\
28 & 0.434 & 300 & 0.521 \\
30 & 0.440 & Rack & 0.550 \\
\hline
\end{tabular}

Table 3.8. Width to Centre Distance Ratio

\begin{tabular}{lc}
\hline \multicolumn{1}{c}{ Type of gear transmission } & $\psi=\mathbf{b} / \mathbf{a}$ \\
\hline $\begin{array}{l}\text { Open type of gearing } \\
\text { Speed reducers (closed type) }\end{array}$ & 0.1 to 0.3 \\
a) High speed 8 to $25 \mathrm{~m} / \mathrm{s}$ & up to 0.3 \\
b) Medium speed 3 to $8 \mathrm{~m} / \mathrm{s}$ & up to 0.6 \\
c) Low speed 1 to $3 \mathrm{~m} / \mathrm{s}$ & up to 1.0 \\
Gear boxes with sliding gears & 0.12 to 0.15 \\
\hline
\end{tabular}


Table 3.9. Allowable Peripheral Speed of Gears

\begin{tabular}{lllll}
\hline & \multirow{2}{*}{$\begin{array}{c}\text { IS quality Preferred } \\
\text { quality }\end{array}$} & \multicolumn{2}{c}{ Speed of gears in m/s } \\
\cline { 4 - 5 } & & & $\begin{array}{l}\text { Cylindrical } \\
\text { gears }\end{array}$ & $\begin{array}{l}\text { Straight bevel } \\
\text { gears }\end{array}$ \\
\hline $\begin{array}{l}\text { High } \\
\text { precision }\end{array}$ & $3 \& 4$ & 4 & Above 15 & Upto 9 \\
Precision & $5 \& 6$ & 6 & $8-15$ & Upto 6 \\
$\begin{array}{l}\text { Medium } \\
\text { Coarse }\end{array}$ & $7,8, \& 9$ & 8 & $1-8$ & Upto 3 \\
\hline
\end{tabular}

\section{Design Calculation}

Standard gear teeth- $20^{\circ}$ stub involute

$\begin{array}{llll}\text { Material } & & =\text { Steel } \\ \text { Poisson's Ratio } & \mu & =0.27 \text { to } 0.30 \\ \text { Young's Modulus } & \mathrm{E} & =2.1^{*} 10^{5} \mathrm{~N} / \mathrm{mm}^{2} \\ & \text { Module } & \mathrm{m} & =10 \\ \text { Speed of pinion } & \mathrm{n}_{1} & =1800 \mathrm{rpm} \\ & \text { Gear ratio } & \mathrm{i} & =1: 3 \\ & \text { Pinion no. of teeth } \mathrm{Z}_{1} & =16 \\ \text { Life of teeth } & \mathrm{L}_{\mathrm{H}} & =20000 \mathrm{hr} \\ & \text { Pressure angle } & (\alpha) & =20^{0} \\ & \text { Power } & \mathrm{P} & =18 \mathrm{KW}\end{array}$

\section{Calculation :-}

Speed of gear $\mathrm{n}_{2}=1800 / 3=600 \mathrm{rpm}$

Gear no. of teeth $Z_{2}=12^{\star} 3=36$

Pitch circle diameter for pinion, $\mathrm{d}_{1}=\mathrm{m}^{\star} \mathrm{z}_{1}=10^{\star} 12=120 \mathrm{~mm}$

Pitch circle diameter for Gear, $\mathrm{d}_{2}=\mathrm{m}^{\star} \mathrm{z}_{2}=10^{\star} 36=360 \mathrm{~mm}$ Center distance of gear \& pinion $\mathrm{a}=\left(\mathrm{z}_{2}+\mathrm{z}_{1}\right) \mathrm{m} / 2=(36+12) 10 / 2=240 \mathrm{~mm}$ Base circle diameter for Pinion, $\mathrm{d}_{\mathrm{b} 1}=\mathrm{d} 1^{*} \cos \alpha=120^{*} \cos 20^{\circ}=112.76 \mathrm{~mm}$ Base circle diameter for Gear, $\mathrm{d}_{\mathrm{b} 2}=\mathrm{d}_{2}{ }^{*} \cos \alpha=360^{*} \cos 20^{\circ}=338.3 \mathrm{~mm}$ Tip circle diameter for pinion, $\mathrm{d}_{\mathrm{a} 1}=\mathrm{d}_{1}+2 \mathrm{~m}=120+2^{\star} 10=140 \mathrm{~mm}$ Tip circle diameter for gear, $\mathrm{d}_{\mathrm{a} 2}=\mathrm{d}_{2}+2 \mathrm{~m}=360+2^{\star} 10=380 \mathrm{~mm}$ Root circle diameter for pinion, $\mathrm{d}_{\mathrm{fl}}=\mathrm{d}_{1}-2^{\star} 1.25 \mathrm{~m}=120-2^{\star} 1.25^{\star} 10=95 \mathrm{~mm}$ Root circle diameter for gear, $\mathrm{d}_{\mathrm{f} 2}=\mathrm{d}_{2}-2^{\star} 1.25 \mathrm{~m}=360-2^{\star} 1.25^{\star} 10=335 \mathrm{~mm}$
Tooth thickness on pitch circle,

$\mathrm{S}=\pi \mathrm{m} / 2=\pi^{\star} 10 / 2=15.71 \mathrm{~mm}$

Clearance $\quad c=0.25 \mathrm{~m}=0.25^{*} 10=2.5 \mathrm{~mm}$

Total Depth, $\quad \mathrm{h}=2.25^{\star} \mathrm{m}=2.25^{\star} 10=22.5 \mathrm{~mm}$

Circular pitch, $\mathrm{p}=\pi \mathrm{m}=\pi^{*} 10=3.14^{*} 10=31.4 \mathrm{~mm}$

Face width, $b=3^{\star} \pi^{*} \mathrm{~m}=3^{*} \pi^{*} 10=94.25 \mathrm{~mm}$

Diametrical pitch $\mathrm{p}_{\mathrm{d}}=\mathrm{z} / \mathrm{d}$ or $1 / \mathrm{m}=1 / 10=0.1$

Pitch line velocity $\mathrm{V}=\pi^{\star} \mathrm{d}_{1}{ }^{*} \mathrm{n}_{1} /\left(60^{\star} 1000\right)=11.31 \mathrm{~m} / \mathrm{sec}$

Ratio of gear width to center distance $\psi=b / a=0.3$

Face width $\mathrm{b}=\psi^{\star} \mathrm{a}=0.3^{\star} 240=72 \mathrm{~mm}$

Pinion life $\mathrm{L}=60 \mathrm{n}_{1} \mathrm{~L}_{\mathrm{H}}=60^{\star} 1800^{\star} 20000$

$$
\begin{aligned}
& =2160000000 \text { revolutions } \\
& =216^{*} 10^{7} \mathrm{rev} .
\end{aligned}
$$

Pinion Torque

$$
\begin{aligned}
\mathrm{M}_{\mathrm{t}} & =\mathrm{P}^{\star} 60 /\left(2 \pi \mathrm{n}_{1}\right)=10000^{\star} 60 /\left(2^{\star} 3.14^{\star} 1800\right) \\
& =95.5 \mathrm{~N} \mathrm{~m}
\end{aligned}
$$

\section{a) Pinion}

1. Material : Case hardening steel is used. Assume surface hardness $55 \mathrm{RC}$ and core hardness greater than $350 \mathrm{BHN}$. (Table 3.6, Table 3.9)

Design stresses

Design bending stress $\sigma_{\mathrm{b}}=\frac{1.4 K \mathrm{kl}}{n K \sigma} \sigma$ $\sigma_{-1}=0.25\left(\sigma_{\mathrm{u}}+\sigma_{\mathrm{y}}\right)+50$ (for cast steel)

$\sigma_{\mathrm{y}}=879 \mathrm{~N} / \mathrm{mm}^{2}$

$\sigma_{-1}=0.25(1050+879)+50=532.25 \mathrm{~N} / \mathrm{mm}^{2}$

$\mathrm{n}=2.5$ (for surface hardened steel)

$\mathrm{K}_{\sigma}=1.5$ (for surface hardened steel)

For steel, surface hardness $>350 \mathrm{BHN}$, core hardness $>350$ BHN and life

$\mathrm{N}$ is $\geq 25^{\star} 10^{7}, \mathrm{~K}_{\mathrm{bl}}=0.7$

$\sigma_{\mathrm{b}}=\frac{1.4 K \boldsymbol{K} l}{n K \sigma} \sigma_{-1}$

$=\left(1.4^{\star} 0.7^{\star} 532.25\right) /\left(2.5^{\star} 1.5\right)=139.1 \mathrm{~N} / \mathrm{mm}^{2}$

Design Contact Stress $\left[\sigma_{c}\right]$

$$
\left[\sigma_{\mathrm{c}}\right]=\mathrm{C}_{\mathrm{R}}{ }^{*} \mathrm{HRC}^{\star} \mathrm{K}_{\mathrm{cl}} \ldots \ldots \ldots \ldots . . \mathrm{N} / \mathrm{mm}^{2}
$$

For surface hardened alloy steel $C_{R}=23$

For steel with surface hardness $>350 \mathrm{BHN}$ and $\mathrm{N} \geq 25^{\star} 10^{7}$, $\mathrm{K}_{\mathrm{cl}}=0.585$

Therefore,

$\left[\sigma_{c}\right]=23^{\star} 55^{\star} 0.585=740.025 \mathrm{~N} / \mathrm{mm}^{2}$

Design Torque $\left[\mathrm{M}_{\mathrm{t}}\right]$

where,

$$
\left[\mathrm{M}_{\mathrm{t}}\right]=\mathrm{k}_{0}^{*} \mathrm{k}^{*} \mathrm{k}_{\mathrm{d}}^{*} \mathrm{M}_{\mathrm{t}}
$$

$\mathrm{K}_{0}=1.5$ for medium shock

$\psi_{\mathrm{P}}=\mathrm{b} / \mathrm{d}_{1}=72 / 120=0.6$ 
Assume IS quality 6 gears (Table 3.2), for pitch line velocity $11.31 \mathrm{~m} / \mathrm{s}$.

$$
\begin{aligned}
& \mathrm{k}=1.03 \\
& \mathrm{k}_{\mathrm{d}}=1.3
\end{aligned}
$$

$\left[\mathrm{M}_{\mathrm{t}}\right]=1.5^{\star} 1.03^{\star} 1.3^{\star} 53.08=191.79 \mathrm{Nm}$

To Calculate The Induced Stresses

$\sigma_{\mathrm{b}}=\frac{\boldsymbol{i}+\mathbf{1}}{\boldsymbol{a} \boldsymbol{m} \boldsymbol{b} \boldsymbol{y}}\left[\mathrm{M}_{\mathrm{t}}\right]$

Form factor for $\mathrm{z}_{1}=16, \mathrm{y}=0.355$ (Table). For stud tooth,

$$
\mathrm{Y}=0.355 / 0.6=0.592)
$$

$\sigma_{\mathrm{b}}=\left[(3+1)\left(191.79^{\star} 1000\right)\right] /\left(240^{\star} 10^{\star} 72^{\star} 0.592\right)=7.5 \mathrm{~N} / \mathrm{mm}^{2}$

$\sigma_{\mathrm{b}}=7.5 \mathrm{~N} / \mathrm{mm}^{2}<\left[\sigma_{\mathrm{b}}\right]=139.1 \mathrm{~N} / \mathrm{mm}^{2}$

$\sigma_{\mathrm{c}}=0.74 \frac{i+1}{a} \sqrt{\frac{i \pm 1}{i b} E[\mathrm{Mt}]}$

$\sigma_{c}=0.74((3+1) / 240) \sqrt{ }\left[\left((3+1) /\left(3^{\star} 72\right)\right)\left(2.1^{\star} 10^{5 *} 191.79^{\star} 10^{3}\right)\right]$

$\sigma_{c}=336.83 \mathrm{~N} / \mathrm{mm}^{2}$

$\sigma_{c}=336.83 \mathrm{~N} / \mathrm{mm}^{2}<\left[\sigma_{\mathrm{c}}\right]=740.025 \mathrm{~N} / \mathrm{mm}^{2}$

The design is satisfactory.

\section{b) Gear}

Gear life $\mathrm{L}=60^{\star} 600^{\star} 20000=720000000 \mathrm{rev}$.

$$
=72^{*} 10^{7} \mathrm{rev} \text {. }
$$

Gear Torque $M_{t}=P^{*} 60 /\left(2 \pi n_{2}\right)=286.48 \mathrm{Nm}$

Material : CS 85, case hardness $55 \mathrm{RC}$ and core hardness greater than $350 \mathrm{BHN}$.

Design stresses

Design bending stress $\sigma_{\mathrm{b}}=\frac{1.4 \mathrm{Kbl}}{n K \sigma} \sigma_{-1}$ $\sigma_{-1}=0.22\left(\sigma_{\mathrm{u}}+\sigma_{\mathrm{y}}\right)+50$ (for cast steel)

$\sigma_{\mathrm{u}}=850 \mathrm{~N} / \mathrm{mm}^{2}$

$\sigma_{\mathrm{y}}=710 \mathrm{~N} / \mathrm{mm}^{2}$

$\sigma_{-1}=0.22(850+710)+50=393.2 \mathrm{~N} / \mathrm{mm}^{2}$

$\mathrm{n}=2.0$ (for case hardened steel)

$\mathrm{K}_{\sigma}=1.2$ (for case hardened steel)

For steel, surface hardness $>350 \mathrm{BHN}$, core hardness $>350$

BHN and life

$\mathrm{N}$ is $\geq 25^{\star} 10^{7}, \mathrm{~K}_{\mathrm{bl}}=0.7$

$\sigma_{\mathrm{b}}=\frac{1.4 K b l}{n K \sigma} \sigma_{-1}$

$=\left(1.4^{\star} 0.7^{\star} 393.2\right) /\left(2.0^{\star} 1.2\right)=160.556 \mathrm{~N} / \mathrm{mm}^{2}$

Design Contact Stress $\left[\sigma_{c}\right]$

$\left[\sigma_{\mathrm{c}}\right]=\mathrm{C}_{\mathrm{R}}{ }^{*} \mathrm{HRC}^{\star} \mathrm{K}_{\mathrm{cl}} \ldots \ldots \ldots \ldots \ldots \mathrm{N} / \mathrm{mm}^{2}$

For case hardened alloy steel $\mathrm{C}_{\mathrm{R}}=28$

For steel with surface hardness $>350 \mathrm{BHN}$ and $\mathrm{N} \geq 25^{\star} 10^{7}$, $\mathrm{K}_{\mathrm{cl}}=0.585$

Therefore,

$\left[\sigma_{c}\right]=28^{\star} 55^{\star} 0.585=900.9 \mathrm{~N} / \mathrm{mm}^{2}$

Design Torque $\left[\mathrm{M}_{\mathrm{t}}\right]$

Where,

$$
\left[\mathrm{M}_{\mathrm{t}}\right]=\mathrm{k}_{0}^{*} \mathrm{k}^{*} \mathrm{k}_{\mathrm{d}}^{*} \mathrm{M}_{\mathrm{t}}
$$

$\mathrm{K}_{0}=1.5$ for medium shock
$\psi_{\mathrm{P}}=\mathrm{b} / \mathrm{d}_{1}=72 / 360=0.2$

assume IS quality 6 gears (Table 1.21), for pitch line velocity $11.31 \mathrm{~m} / \mathrm{s}$.

$$
\begin{aligned}
& \mathrm{k}=1.0 \\
& \mathrm{k}_{\mathrm{d}}=1.3
\end{aligned}
$$

$\left[\mathrm{M}_{\mathrm{t}}\right]=1 \cdot 5^{\star} 1.0^{\star} 1.3^{\star} 286.48=558.636 \mathrm{Nm}$

To Calculate The Induced Stresses

$\sigma_{\mathrm{b}}=\frac{\boldsymbol{i}+\mathbf{1}}{\boldsymbol{a} \boldsymbol{m} \boldsymbol{b} \boldsymbol{y}}\left[\mathrm{M}_{\mathrm{t}}\right]$

(Form factor for $\mathrm{z}_{1}=48, \mathrm{y}=0.475$ (Table For stud tooth,

$$
\mathrm{Y}=0.475 / 0.2=2.375)
$$

$\sigma_{\mathrm{b}}=\left[(3+1)\left(558.636^{\star} 1000\right)\right] /\left(240^{\star} 10^{\star} 72^{\star} 2.375\right)$

$$
=5.445 \mathrm{~N} / \mathrm{mm}^{2}
$$

$\sigma_{\mathrm{b}}=5.445 \mathrm{~N} / \mathrm{mm}^{2}<\left[\sigma_{\mathrm{b}}\right]=160.556 \mathrm{~N} / \mathrm{mm}^{2}$

$\sigma_{\mathrm{c}}=0.74 \frac{i+1}{a} \sqrt{\frac{i \pm 1}{i b} E[\mathrm{Mt}]}$

$\sigma_{c}=0.74((3+1) / 240) \sqrt{ }\left[\left((3+1) /\left(3^{\star} 72\right)\right)\left(2.1^{\star} 10^{5 \star} 558.636^{\star} 10^{3}\right)\right]$

$\sigma_{c}=574.854 \mathrm{~N} / \mathrm{mm}^{2}$

$\sigma_{c}=574.854 \mathrm{~N} / \mathrm{mm}^{2}<\left[\sigma_{c}\right]=901 \mathrm{~N} / \mathrm{mm}^{2}$

The design is satisfactory.

\section{Dynamic Load}

The level of vibration and noise of the gear pairs is in correlation with the characteristics of dynamic load. Calculation of dynamic loads and determination of their variation during a mesh cycle for spur gears pairs has been considered a major aspect of gear design. An analytical model that covers the main influence factors with sufficient accuracy is not currently available.

This paper presents an analytical approach used in order to predict the characteristics of dynamic loads by considering the time-varying mesh stiffness and variable tooth profile errors. The calculus procedure of the mesh stiffness is found by using an exact analytical model.

\subsection{Dynamic Load Procedure}

Dynamic load

$\mathrm{F}_{\mathrm{d}}=2 e_{/} \boldsymbol{t}_{\mathrm{v}}\left(\boldsymbol{k m} \boldsymbol{\mathrm { e }}_{\mathrm{e}}\right) \ldots$

eq. 1

Here e is the sum of the errors, inches, of the two mating teeth, $\mathrm{t}$ is the time, seconds, during which the error is acting, $\mathrm{k}$ is the spring constant, pounds per inch, of the two mating teeth, and $m_{e}$ is the effective mass, $1 b \sec ^{2} /$ in., of the two gears.

Time $t$ for the passage of a single pitch, then is $\mathrm{t}=60 /\left(\mathrm{n}_{1} \mathrm{~N}_{1}\right) \sec \ldots$

eq. 2 
$\mathrm{K}=\mathrm{b} / 9\left[\left(\mathrm{E}_{1} \mathrm{E}_{2}\right) /\left(\mathrm{E}_{1}+\mathrm{E}_{2}\right)\right] \ldots \ldots$.

eq. 3

Where $\mathrm{E}_{1}$ and $\mathrm{E}_{2}$ are the module of elasticity for the materials of the two gears.

When both gear \& pinion are steel, $\mathrm{E}_{1}=\mathrm{E}_{2}=30,000,000$ psi, $=2.1^{*} 10^{5} \mathrm{~N} / \mathrm{mm}^{2}$ the value of $\mathrm{k}$ for a meshing pair becomes $\mathrm{K}=1,670,000 \mathrm{~b} \mathrm{lb} / \mathrm{in} .=11500 \mathrm{~b} \mathrm{~N} / \mathrm{mm}$

When one gear is steel, $\mathrm{E}_{1}=30,000,000$ psi and the other gear is cast iron or bronze, $\mathrm{E}_{2}=16,000,000 \mathrm{psi},=1.1^{\star} 10^{5} \mathrm{~N} / \mathrm{mm}^{2}$

$\mathrm{K}=1,160,000 \mathrm{~b} \mathrm{lb} / \mathrm{in} .=8000 \mathrm{~b} \mathrm{~N} / \mathrm{mm}$

$\mathrm{b}=$ width of face of the gears.

The moment of inertia of a gear can be found by approximating it as a solid circular cylinder of diameter equal to the pitch diameter of the gear, and axial length equal to the face width of the teeth. The mass moment of inertia I, fig.3$1(\mathrm{a})$, then is

$$
I=\mathrm{m}\left(\mathrm{r}^{2} / 2\right)
$$

Where $m$ is the mass, with dimensions of $1 \mathrm{~b} \mathrm{sec} / \mathrm{in}$. as found from Newton's second law of motion,

mass $=$ force/acceleration. Mass is thus equal to the weight divided by the gravitational constant $\mathrm{g}$.

A concentrated mass $m$ ', located at the pitch circle, has a moment of inertia $I^{\prime}$ about the axis of

$$
I^{\prime}=m^{\prime} \mathrm{r}_{2}
$$

Suppose $m^{\prime}$ were of such size as to make $I$ and $I^{\prime}$ equal. Then

$$
m^{\prime}=m^{\star} 1 / 2
$$

The dynamical system of the two gears is considered as the masses $m_{1}^{\prime}$ and $m_{2}$

Concentrated at the pitch circles connected by a spring comprising the two teeth. For Such a system, the effective mass $m_{\mathrm{e}}$ is given by the following equation.

$1 / m_{\mathrm{e}}=1 / m_{1}^{\prime}+1 / m_{2}^{\prime} \ldots \ldots$.

Mass $m_{1}^{\prime}$ of the pinion is equal to

$m_{1}^{\prime}=m_{1}(1 / 2)=\left(\pi \mathrm{r}_{1}^{2} \mathrm{~b} \gamma_{1}\right) /(2 \mathrm{~g}) \ldots \ldots$

where $\gamma_{1}$ is the weight per cubic inch of the material.

Mass $m_{2}^{\prime}$ of the gear is given by a similar equation.

$m_{2}^{\prime}=m_{2}(1 / 2)=\left(\pi r_{2}^{2} \mathrm{~b} \gamma_{2}\right) /(2 \mathrm{~g}) \ldots \ldots$.

where $\gamma_{2}$ is the weight per cubic inch of the material composing the gear.

Substitution into Eq.1 gives

$1 / m_{\mathrm{e}}=2 \mathrm{~g} /(\pi \mathrm{b})\left[\left(1 /\left(\mathrm{r}_{1}^{2} \gamma_{1}\right)\right)+\left(1 /\left(\mathrm{r}_{2}^{2} \mathrm{~b} \gamma_{2}\right)\right)\right] \ldots \ldots \ldots$.

\subsection{Design Equations for Dynamic Load}

steel pinion - steel gear .here $\gamma_{1}=\gamma_{2}=0.283 \mathrm{lb} / \mathrm{in}^{3}=$ $0.0000768 \mathrm{~N} / \mathrm{mm}^{3}$ and acceleration ' $\mathrm{g}$ ' due to gravity is taken as $386 \mathrm{in} . / \mathrm{sec}^{2} .=9806.6 \mathrm{~mm} / \mathrm{sec}^{2}$ Equation (7) becomes
$1 / \mathrm{m}_{\mathrm{e}}=\frac{2 * 9806.6}{\pi b 0.0000768}\left(\left(1 / \mathrm{r}_{1}^{2}\right)+\left(1 / \mathrm{r}_{2}^{2}\right)\right)$

$$
=\frac{81,270,000}{b}\left(\left(\mathrm{r}_{1}{ }^{2}+\mathrm{r}_{2}{ }^{2}\right) /\left(\mathrm{r}_{1}{ }^{2} \mathrm{r}_{2}{ }^{2}\right)\right) \mathrm{mm} / \mathrm{N} \mathrm{sec}{ }^{2}
$$

At all values substitute in eq. 1

$\mathrm{F}_{\mathrm{d}}=2 e / t \mathrm{v}(\mathrm{km})$

$=\left(e^{\star} n_{1}{ }^{*} N_{1}{ }^{*} b^{*} r_{1}^{*} r_{2}\right) /\left(2520 \sqrt{ }\left(r_{1}^{2}+r_{2}^{2}\right)\right)$ Newton's

Steel pinion - cast iron gear . hear $\gamma_{1}=0.283 \mathrm{lb} / \mathrm{in}^{3}$, and $\gamma_{2}=$ $0.256 \mathrm{lb} /$ in. $^{3}$ or $0.0000695 \mathrm{~N} / \mathrm{mm}^{3}$

$1 / \mathrm{m}_{\mathrm{e}}=\left(2 \mathrm{~g} / \pi \mathrm{b} \gamma_{2}\right)\left(\left(\mathrm{r}_{1}^{2}+\left(\gamma_{2} / \gamma_{1}\right) \mathrm{r}_{2}^{2}\right) /\left(\mathrm{r}_{1}^{2} \mathrm{r}_{2}^{2}\right)\right)$

$=\frac{89840,000}{b}\left(\left(\mathrm{r}_{1}^{2}+0.9 \mathrm{r}_{2}^{2}\right) /\left(\mathrm{r}_{1}^{2} \mathrm{r}_{2}^{2}\right)\right)$

At all values substitute in eq. 1

$\mathrm{F}_{\mathrm{d}}=2 e / \mathrm{t} \mathrm{v}\left(\mathrm{km} \mathrm{e}_{\mathrm{e}}\right)$

$=\left(e^{\star} n_{1}{ }^{\star} N_{1}{ }^{*} b^{\star} r_{1}^{*} r_{2}\right) /\left(3180 \sqrt{ }\left(r_{1}^{2}+0.9 r_{2}^{2}\right)\right) \mathrm{N}$

\subsection{Dynamic Load}

\section{a) Process 1}

Dynamic Load

$\mathrm{F}_{\mathrm{d}}=2 e / \mathrm{t} v\left(\boldsymbol{k m} \mathrm{e}_{\mathrm{e}}\right)$

$=\left(\mathrm{e}^{\star} \mathrm{n}_{1}{ }^{\star} \mathrm{N}_{1}{ }^{*} \mathrm{~b}^{\star} \mathrm{r}_{1}{ }^{\star} \mathrm{r}_{2}\right) /\left(2520 \sqrt{ }\left(\mathrm{r}_{1}{ }^{2}+\mathrm{r}_{2}{ }^{2}\right)\right)(\mathrm{N}=\mathrm{z}$ no. of teeth $)$

$\mathrm{e}=0.0048+0.0050=0.0098$ in. $=0.2489 \mathrm{~mm}$

$(1 \mathrm{in}=25.4 \mathrm{~mm})$

$\mathrm{F}_{\mathrm{d}}=0.2489^{\star} 1800^{\star} 16^{\star} 72^{\star} 60^{\star} 180 /\left(2520 \sqrt{ }\left(60^{2 \star}+180^{2}\right)\right)$

$=11657.9 \mathrm{~N}$

$\mathrm{F}_{\mathrm{w}}=\mathrm{d}_{1} \mathrm{bQ} K$

Where, $d_{1}=120 \mathrm{~mm}, b=72 \mathrm{~mm}$

$\mathrm{Q}=2 \mathrm{z}_{2} /\left(\mathrm{z}_{1}+\mathrm{z}_{2}\right)$

$=2^{\star} 48 /(16+48)=1.5$

$K=651 \mathrm{psi}=4.48842 \mathrm{~N} / \mathrm{mm}^{2}$

$\mathrm{F}_{\mathrm{w}}=120^{\star} 72^{\star} 1.5^{\star} 4.48842$

$=58.1699 \mathrm{KN}$

$\mathrm{F}_{\mathrm{t}}=\mathrm{F}_{\mathrm{w}}-\mathrm{F}_{\mathrm{d}}$

$=58.1699-11.679$

$=46.4909 \mathrm{KN}$

\section{b) Process 2}

Buckingham's Dynamic Load

$\mathrm{F}_{\mathrm{d}}=\mathrm{F}_{\mathrm{t}}+\left[0.164 \mathrm{~V}_{\mathrm{m}}\left(\mathrm{c}^{\star} \mathrm{b}+\mathrm{F}_{\mathrm{t}}\right) /\left[0.164 \mathrm{~V}_{\mathrm{m}}+1.486 \sqrt{ }\left(\mathrm{c}^{\star} \mathrm{b}+\mathrm{F}_{\mathrm{t}}\right)\right]\right]$

Where, $\mathrm{v}_{\mathrm{m}}=$ pitch line velocity, $=11.31 \mathrm{~m} / \mathrm{s}$ or $678.6 \mathrm{~m} / \mathrm{min}$ $\mathrm{V}_{\mathrm{m}}=678.6 \mathrm{~m} / \mathrm{min}$

$\mathrm{b}=$ face width $=72 \mathrm{~mm}=0.072 \mathrm{~m}$

$\mathrm{c}=$ factor depending on machining error $=12300 \mathrm{e}$

$=12300^{*} 0.03=369$

$\mathrm{F}_{\mathrm{t}}=$ transmitted load, kgf

$\mathrm{F}_{\mathrm{t}}=\mathrm{HP}^{\star} 75 / \mathrm{v}_{\mathrm{m}}$

$=24.14^{\star} 75 / 11.31$ 


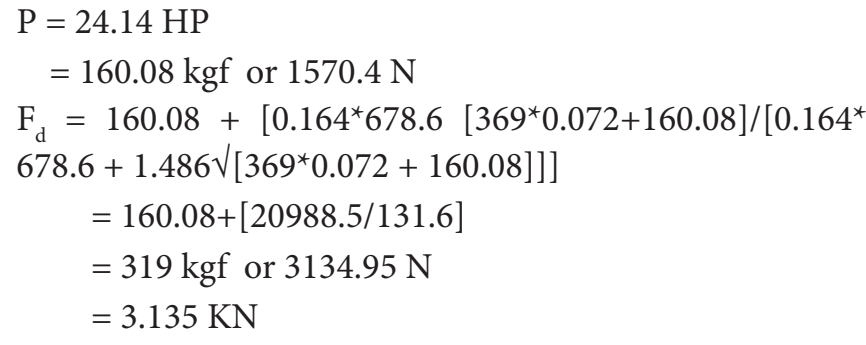

\section{c) Process 3}

Lewis Equation of dynamic load

$\mathrm{F}_{\mathrm{d}}=\mathrm{F}_{\mathrm{t}}^{*} \mathrm{C}_{\mathrm{v}}$

$\mathrm{F}_{\mathrm{t}}=\mathrm{HP}^{\star} 75 / \mathrm{v}_{\mathrm{m}}$

$\mathrm{v}_{\mathrm{m}}=11.31 \mathrm{~m} / \mathrm{s}$

$\mathrm{P}=24.14 \mathrm{hp}$

$\mathrm{C}_{\mathrm{v}}=\left(6+\mathrm{v}_{\mathrm{m}}\right) / 6$

$=(6+11.31) / 6=2.885$

$\mathrm{F}_{\mathrm{t}}=24.14^{\star} 75 / 11.31$

$=160.08 \mathrm{kgf}$

$\mathrm{F}_{\mathrm{d}}=160.08^{\star} 2.885$

$=461.831 \mathrm{kgf}$

$=4530.6 \mathrm{~N}$ or $4.531 \mathrm{KN}$

\section{Conclusions}

Design of spur gears using steel-steel alloys is done and the dynamic loads on spur gears are calculated by using complete study of literature and reporting.
In phase -2 the design of spur gears using Sintered materials is yet to be done. The dynamic load characteristic is to be calculated manually then the model is created in CAD software's.

An analytical investigation of the dynamic loads fluctuation in spur gears is presented. The dynamic model permits to predict the individual tooth load characteristics during the meshing cycle.

\section{References}

1. Winter H. (1961). Gear Tooth Strength of Spur Gears', British Gear Manufacturers Association.

2. A.S.M.E (1931). Dynamic Loads on Gear Teeth.

3. http://nptel.iitm.ac.in/courses/IIT-MADRAS/Machine Design_II/pdf/2_7.pdf

4. Vedmar L, Henriksson B (1998). A general approach for determiningdynamicforcesinSpurgears, JournalofMechanical Design, Transaction of the ASME, 593-598.

5. Prabhu T J (2005). Design of Transmission Elements,

6. Maitra G M (1989). Handbook of Gear Design,1-40

7. Lingaiah K (2007). Machine Design Data Handbook, vol 1.

8. P.S.G. College of Technology (1988). Design Data Book,

9. Merrit H E (1992). Gear Engineering, 15-53,90 Dr. Ulrich Kissling, Effects of Profile corrections on peak - to - peak transmission error, Gear Technology.

10. Kiss Soft (2008). Gear Optimizing with advanced calculation method. www.kisssoft.ch/english/downloads/pdf/Gear_ Optimizing 